\title{
A Normalized Congruent Matching Area Method for the Correlation of Breech Face Impression Images
}

\author{
Zhe Chen $^{1,2}$, John Song ${ }^{2}$, Wei Chu ${ }^{2}$, Mingsi Tong ${ }^{1}$, and XueZeng Zhao ${ }^{1}$ \\ ${ }^{1}$ School of Mechatronics Engineering, \\ Harbin Institute of Technology, \\ Harbin 150001, China \\ ${ }^{2}$ National Institute of Standards and Technology, \\ Gaithersburg, MD 20899 USA \\ zhe.chen@nist.gov \\ jun-feng.song@nist.gov \\ wei.chu@nist.gov \\ mingsi.tong@gmail.com \\ zhaoxz@hit.edu
}

\begin{abstract}
The congruent matching cells (CMC) method was invented at the National Institute of Standards and Technology (NIST) for firearm evidence identification and error rate estimation. The CMC method divides the correlated image pairs into cells and uses four parameters to quantify topography similarity and pattern congruency of the correlated cell pairs in firearm breech face impressions on fired cartridge cases. A preliminary conservative numerical identification criterion of $C=6 \mathrm{CMCs}$ was suggested for identifying images of cartridge cases fired from the same firearm. The CMC method was validated by correlations using both three-dimensional (3D) topography images and two-dimensional (2D) optical images from a set of 40 cartridge cases fired from a firearm set composed of 10 consecutively manufactured pistol slides. However, in the original CMC method, due to the difference in the effective data area of the correlated cells, final CMCs obtained from an image pair presented different data quantity (or validity level), and thus the empirical criterion $C=6 \mathrm{CMCs}$ did not remain optimal for identification when the correlated cell size changed. In this study, a normalized congruent matching area (NCMA) method that considers the difference in the data area in each correlated cell pair was developed. Based on the NCMA method, an optimal range of cell sizes for breech face identification with granular characteristics was determined. A binomial model was used to fit the known nonmatching NCMA probability distribution $\Psi_{\mathrm{NCMA}}$, and a beta-binomial model was used to fit the known matching NCMA probability distribution $\Phi_{\mathrm{NCMA}}$. An experimental improvement in the normalized identification criterion $C$ of around $6 \%$ was observed in the validation tests when the cell sizes were in the optimal range.
\end{abstract}

Key words: cartridge case; congruent matching cells (CMC); effective data area; firearm evidence identification; forensics; impression evidence; optimal cell size.

Accepted: June 30, 2018

Published: August 6, 2018

https://doi.org/10.6028/jres.123.015

\section{Background}

A "firearm signature" [1] is a special kind of toolmark left by the related parts of the firearm (gun barrel, firing pin, breech face, and ejector) that make forcible contact with bullets and cartridge cases. For 
breech face signatures left on fired cartridge cases, one vital fact is that not all regions of the primer make optimal contact with the breech face of the firearm. For automated correlation systems, these "invalid" correlation regions may reduce the accuracy of the overall correlation [2].

In 2012, a new method for firearm identifications, named the congruent matching cells (CMC) method, was invented at the National Institute of Standards and Technology (NIST) [3]. The CMC method divides the correlated image pairs of the impressed toolmark into cells and uses four parameters to quantify the topography similarity and pattern congruency of the cell pairs. This process is based on the principle of discretization and can reduce the effects of "invalid regions." A numerical identification criterion for the required number of CMC pairs of $C=6 \mathrm{CMCs}$ was suggested as a conservative preliminary identification criterion for identifying topography images of cartridge cases fired from the same firearm [3]. Initial tests using both three-dimensional (3D) topography images [4] and two-dimensional (2D) optical images [5] from a set of 40 cartridge cases ejected from firearms with 10 consecutively manufactured pistol slides showed that all the image pairs were correctly identified without any false identification or false exclusion $[4,5]$.

However, the numerical criterion of $C=6 \mathrm{CMCs}$ is not always optimal, because the result of CMC scores is greatly influenced by the actual number of cell pairs included in the correlation calculation [4]. This number is determined primarily by the cell size. Additionally, the breech face impression resembles a doughnut shape (see Fig. 1). The actual size of the breech face impression is different from firing to firing, due to the different structures of breech and firing conditions. This leads to differences in effective cell numbers between different sample pairs, and a difference in the validity level of CMCs in similar sample pairs. To establish a robust identification criterion for different cell sizes, numbers, and validity levels, and to define an optimal range for the cell size, a normalized congruent matching area (NCMA) method is proposed. In this paper, the concept of the CMC method is reviewed, and the NCMA method is introduced in Sec. 2. The validation test is conducted, and the optimal cell size is determined in Sec. 3. Test results and experimental robust identification criteria for the optimal cell size ranges are shown in Sec. 4, followed by conclusions in Sec. 5.

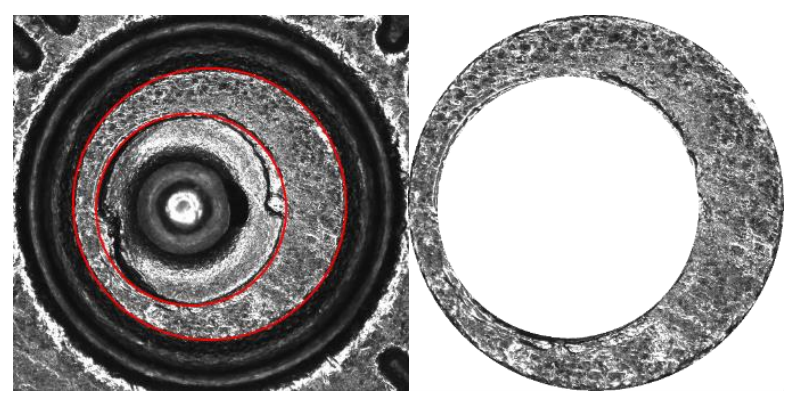

Fig. 1. Optical image of a breech face impression obtained from a cartridge case. The image has a number of features in common, including the firing pin image in the center (left). The doughnut-shaped breech face impression image (right) was extracted for study. The outside diameter of the extracted breech face impression is about $3.8 \mathrm{~mm}$.

\section{Basic Concept}

\subsection{Congruent Matching Cells Method}

Continuing the terminology from our previous research in firearms identification [3, 6], a region of the surface topography on the fired cartridge case is termed a "valid correlation region" if it contains individual characteristics [1] of the firearm signature that can be used effectively for identification. Conversely, a region of the surface topography that does not contain individual characteristics of the firearm signature is 
termed an "invalid correlation region" and should be eliminated from correlation. An invalid correlation region can occur, for example, due to insufficient contact between the firearm surface and the bullet or cartridge case during firing [7].

If two toolmark topographies A and B, originating from the same firearm, are registered at their common maximum correlation location, the cell pairs located in the common valid correlation regions of $\mathrm{A}$ and $\mathrm{B}$ will be characterized by $[3,6]$ :

1. high topography similarity, quantified by the normalized areal cross correlation function maximum $A C C F_{\max }[8]$,

2. similar registration angle $\theta$ for the correlated cell pairs in topographies $\mathrm{A}$ and $\mathrm{B}$, and

3. similar $x-y$ spatial distribution patterns for the cell arrays in topographies A and B.

On the other hand, if the registered cell pairs are in the invalid correlation regions of $\mathrm{A}$ and $\mathrm{B}$, or if they originate from different firearms, their $A C C F_{\max }$ values would be relatively low, and their cell arrays would show significant variation in their $x-y$ distribution patterns and registration angles $\theta$.

The CMCs can be determined by four identification parameters $A C C F_{\max }, \theta, x$, and $\mathrm{y}$, with the corresponding thresholds: $T_{\mathrm{ACCF}}, T_{\theta}, T_{\mathrm{x}}$, and $T_{\mathrm{y}}$. Cell pairs can be considered as CMCs when their correlation values, $A C C F_{\max }$, exceed $T_{\mathrm{CCF}}$, and when their registration angle $\theta$ and registration positions $x-y$ fall within the thresholds $T_{\theta}$ and $T_{\mathrm{x}}-T_{\mathrm{y}}$, respectively. Inspired by the numerical identification criterion of the consecutively matching striae (CMS) method developed by Biasotti and Murdock [9], the initial numerical identification criterion for the $\mathrm{CMC}$ method was suggested as $C=6 \mathrm{CMCs}$ for testing; i.e., two images are concluded to be a "match" when the number of CMC pairs between them is no less than 6 . This initial criterion is undergoing refinement by experimental studies that estimate the known matching (KM) and known nonmatching (KNM) CMC score distributions of different firearm and ammunition brands. For example, a criterion value can be chosen by the KNM distribution when an acceptable estimated false positive error rate is obtained. Alternatively, some quantitative expression for the weight of the evidence associated with the observed number of CMCs can be generated by distributions, such as a score-based random match probability or likelihood ratio.

The CMC method has been validated by different sets of topography measurements [4], by optical images [5], and by different correlation programs and statistical models [7].

\subsection{Normalized Congruent Matching Area Method}

Figure 2 shows the CMC distribution on KM image pair \#19 vs. \#20 (Fadul data set [10]) at different cell sizes. The same image pair generates $24 \mathrm{CMCs}$ at a cell size of $(75 \times 75)$ pixels (Fig. 2a) and $12 \mathrm{CMCs}$ at a cell size of $(100 \times 100)$ pixels (Fig. $2 \mathrm{~b}$ ), with a nominal pixel spacing of $6.25 \mu \mathrm{m}$, using the same CMC threshold values $T_{\mathrm{CCF}}=45 \%, T_{\theta}=3^{\circ}, T_{\mathrm{x}}=T_{\mathrm{y}}=20$ pixels. The considerable difference in the numerical CMC scores indicates that the comparison results are influenced by the cell size. Due to the irregular doughnut shape of the breech face images, some cells contain (or cover) most of the image pixels, as seen in the cell labeled A5 (Fig. 2a), while others only contain (or cover) less than $50 \%$ of the image pixels, for example, cell A19 (Fig. 2a). In the CMC method, however, both A5 and A19 are regarded as CMCs and contribute equally to the final comparison results without considering a weighting function (or validity level) due to the difference in data quantity. 

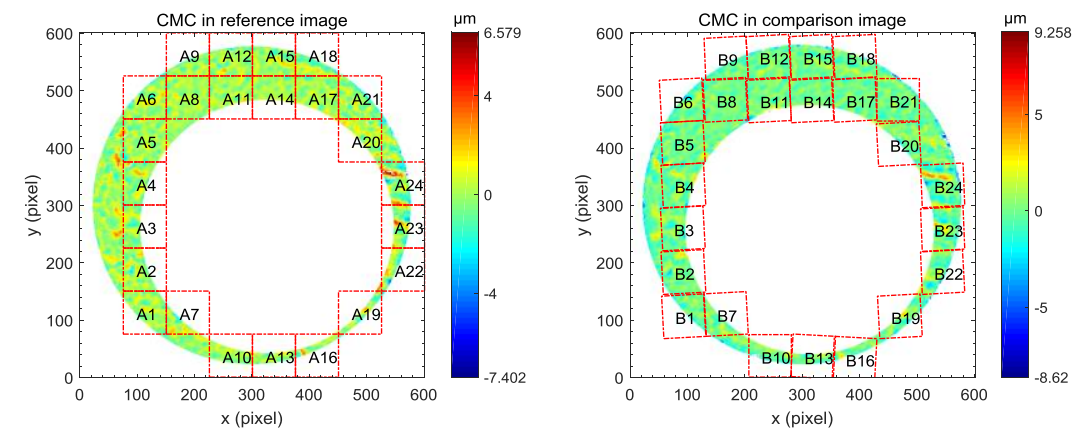

(a) Cell size of $(75 \times 75)$ pixels $(6.25 \mu \mathrm{m}$ per pixel)
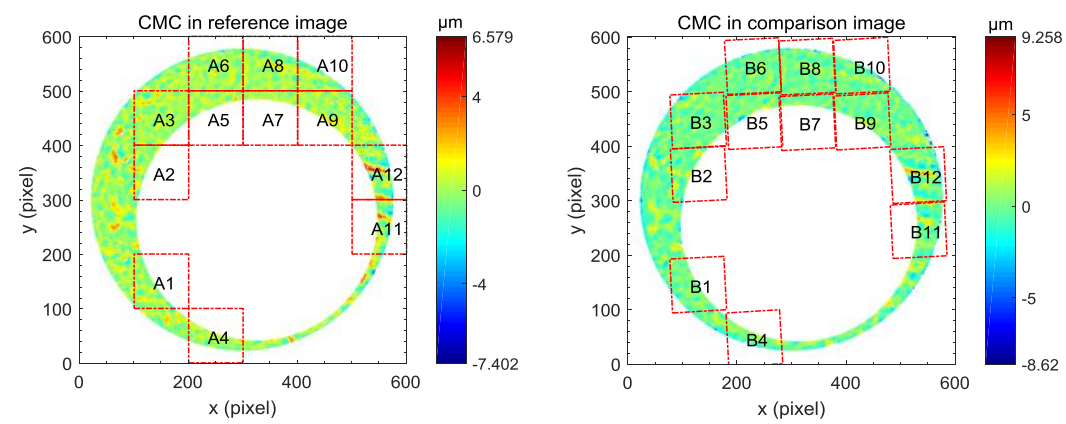

(b) Cell size of $(100 \times 100)$ pixels $(6.25 \mu \mathrm{m}$ per pixel $)$.

Fig. 2. CMC distribution on KM image pair \#19 vs. \#20 (Fadul data set [10]) at different cell sizes. The same image pair generates 24 CMCs at a cell size of $(75 \times 75)$ pixels $(a)$ and 12 CMCs at a cell size of $(100 \times 100)$ pixels $(b)$.

Figure 3 shows a typical CMC distribution on KNM image pair \#12 vs. \#29 (Fadul data set [10]) at a cell size of $(75 \times 75)$ pixels. The pixel spacing and CMC threshold values are the same as that in Fig. 2. In our previous studies $[4,5,7]$, the CMCs on KNM image pairs are most likely to appear at the edges of the breech face impression, as shown in Fig. 3. Cells on the edges contain fewer informative pixels, which may contribute to their being incorrectly identified as CMCs. Therefore, a CMC at the edge should be considered with less weight (or lower validity level) than those located in the middle of the impression with full data quantity (or high validity level). In a study of the identification of degraded land impressions, Eric Hare et al. found that, when the proportion of the land that is recovered is below $50 \%$, the accuracy of the land impression comparison is low [11].
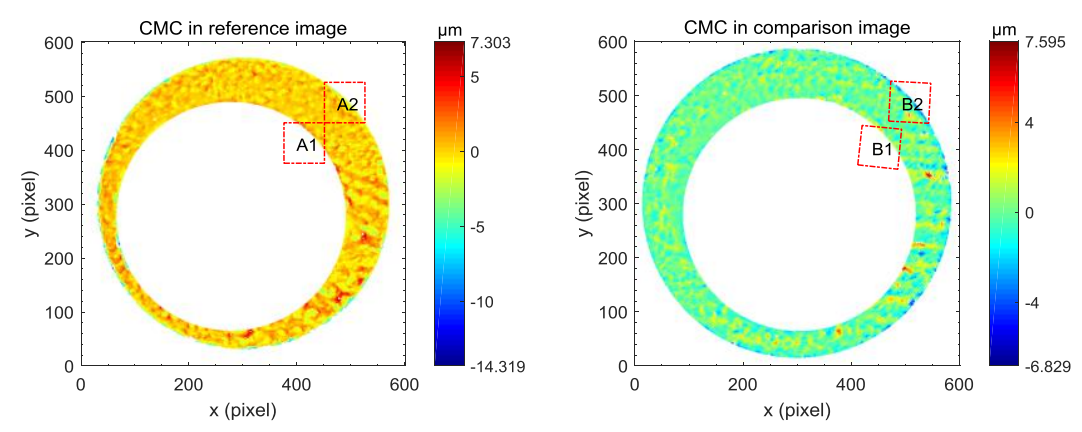

Fig. 3. CMC distribution on KNM pair \#12 vs. \#29 (Fadul data set [10]) at a cell size of $(75 \times 75)$ pixels $(6.25 \mu \mathrm{m}$ per pixel). CMCs on KNM image pairs are most likely to appear at the edges of the breech face impression. 
The NCMA method is thus proposed based on the difference in data quantity in the cells. The NCMA value of a correlated image pair is defined as:

$$
\mathrm{NCMA}=\frac{\text { Congruent matching area }}{\text { Whole breech face area }}=\frac{\sum_{i=1}^{M} D Q_{\mathrm{c}}^{i}}{D Q_{\mathrm{w}}} \times 100 \%
$$

where $M$ is the total number of CMCs identified in the image pair;

$D Q_{\mathrm{c}}^{i}$ is the data quantity in the CMC area of the reference image with serial number $i$; and

$D Q_{\mathrm{w}}$ is the data quantity represented by the number of pixels in the whole reference image.

By this NCMA transformation process, the 24 CMCs in Fig. 2a are transformed to $80.5 \%$ NCMA, which indicates that $80.5 \%$ of the breech face area in the reference image can be considered as "congruent matching" with the comparison image. Similarly, the two CMCs in Fig. 3 are transformed to 5.0 \% NCMA. If the total cell number $N$ is adequate, which means the cell size is reduced to a sufficiently small area for high correlation accuracy, but the area is still large enough to hold topography features, then the NCMA value should hardly change with the cell size, theoretically. For example, the $12 \mathrm{CMCs}$ in Fig. $2 \mathrm{~b}$ are transformed to $61.1 \%$ NCMA, but, compared with the large difference in CMC numbers between Figs. 2a and $2 \mathrm{~b}$ ( 24 to $12,50 \%$ decrease), the NCMA value between them does not change as much (80.5 \% to $61.1 \%$, about $25 \%$ decrease).

\section{Validation Tests and Optimal Range of Cell Size}

A validation test was conducted to find the optimal range of the cell size based on the above discussion. The NCMA correlations were conducted on a set of breech face impressions from the Fadul data set [10], which was created at the Miami-Dade Crime Laboratory for a study of microscopic visual firearm evidence comparisons by firearm examiners. The study sample set contains 40 cartridge cases ejected from Ruger P95PR ${ }^{1}$ pistols with 10 consecutively manufactured slides. Comparisons involving a population of consecutively manufactured firearm parts represent a challenging scenario for accurately identifying bullets or cartridge cases as being fired or ejected from the same firearm. Consecutively manufactured parts can have similar topographic features arising from temporary imperfections in the manufacturing process, such as a worn tool. The presence of these subclass characteristics can be misinterpreted by an examiner as individualizing and possibly lead to false identifications [1].

The breech face impression topographies on the cartridge cases were measured using a scanning disk confocal microscope [12]. The original images have a nominal image area of about $3.8 \mathrm{~mm} \times 3.8 \mathrm{~mm}$ or approximately $(1200 \times 1200)$ pixels with a nominal pixel spacing of $3.125 \mu \mathrm{m}$. The images were trimmed to remove impression edge areas with strong roll-off, caused in part by firing pin drag marks and firing pin aperture shear. Then, the images were decimated to a pixel spacing of $6.25 \mu \mathrm{m}$ to increase the correlation speed. Finally, the images were band-pass filtered using the second-order robust Gaussian regression filter to reduce noise with short wavelengths and to attenuate waviness and form with long wavelengths, thus highlighting potentially individual characteristics. The short wavelength cutoff was $16 \mu \mathrm{m}$, and the long wavelength cutoff was $250 \mu \mathrm{m}$. Figure 4 shows a typical impression topography for this data set after trimming and filtering, which can be generally characterized as possessing granular breech face toolmark impression characteristics.

${ }^{1}$ Certain commercial equipment, instruments, or materials are identified in this paper to specify the experimental procedure adequately. Such identification is not intended to imply recommendation or endorsement by the National Institute of Standards and Technology, nor is it intended to imply that the materials or equipment identified are necessarily the best available for the purpose. 


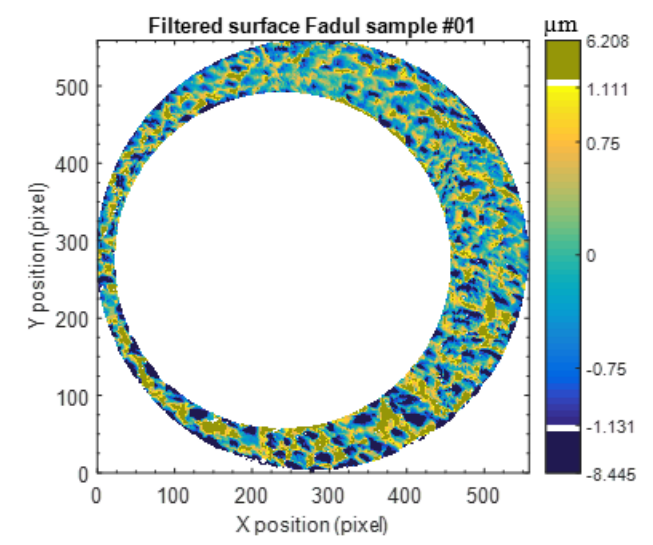

Fig. 4. Topography features with granular characteristics of the filtered breech face impression for Fadul sample \#1 of the Fadul data set [10].

For the NCMA method, the correlation was conducted with the cell size chosen from $(15 \times 15)$ pixels to $(120 \times 120)$ pixels, with an increment of 5 pixels, and the corresponding nominal cell number $\left(N_{\text {nom }}\right)$ ranged from $(38 \times 38)$ to $(5 \times 5)$. Cells containing insufficient data points (in our test, cells with less than $15 \%$ of their pixels representing measured data) were excluded from the analysis. The rotation angle range was $\pm 30^{\circ}$ with $3^{\circ}$ increments. The thresholds $T_{\theta}, T_{\mathrm{x}}$, and $T_{\mathrm{y}}$ were set as $T_{\theta}=3^{\circ}, T_{\mathrm{x}}=20$ pixels, and $T_{\mathrm{y}}=20$ pixels, respectively (the thresholds $T_{\mathrm{x}}$ and $T_{\mathrm{y}}$ were reduced to 15 pixels at the cell size of [20 $\times 20$ ] pixels and to 10 pixels at the cell size of $[15 \times 15]$ pixels). As shown in Fig. 5, the closeness between the topography similarity of the KM and KNM image pairs is demonstrated by the overlap of $A C C F_{\max }$ distributions for the KM and KNM cell pairs. This closeness changes at different cell sizes and can be quantified by two distribution parameters: the false cell similarity identification frequency $f_{1(\mathrm{ACCF})}$ and the false cell similarity exclusion frequency $f_{2(\mathrm{ACCF})}[6]$ :

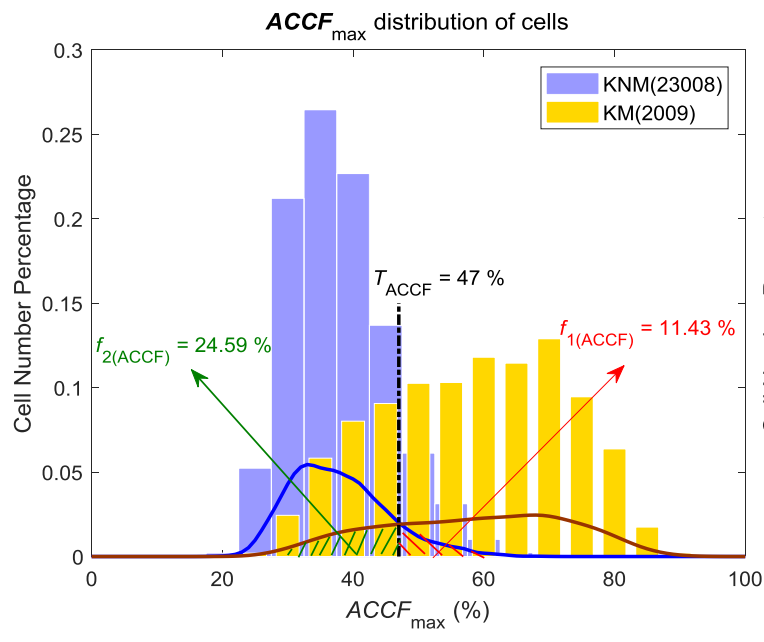

(a) Cell size: $(75 \times 75)$ pixels $(6.25 \mu \mathrm{m}$ per pixel).

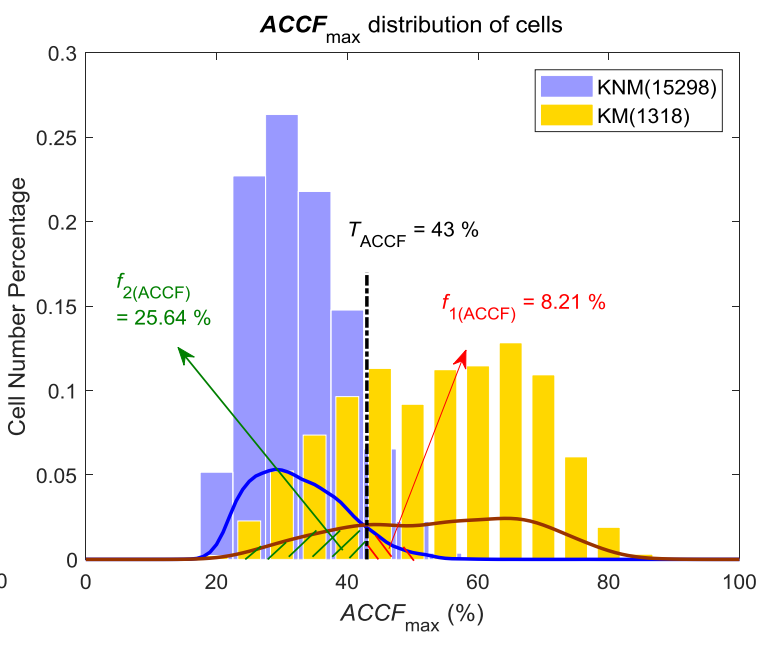

(b) Cell size: $(100 \times 100)$ pixels $(6.25 \mu \mathrm{m}$ per pixel).

Fig. 5. $A C C F_{\max }$ distribution of cell pairs in KM and KNM image correlations for Fadul data set [10] at different cell sizes, where the optimal $T_{\mathrm{ACCF}}$ value was chosen at the intersection point of the two fit distributions. 


$$
\begin{gathered}
f_{1(\mathrm{ACCF})}=\frac{\text { Number of KNM cell pairs for which } A C C F_{\max } \geq T_{\mathrm{ACCF}}}{\text { Total number of evaluated KNM cell pairs }} \\
f_{2(\mathrm{ACCF})}=\frac{\text { Number of KM cell pairs for which } A C C F_{\max }<T_{\mathrm{ACCF}}}{\text { Total number of evaluated KM cell pairs }}
\end{gathered}
$$

These estimated cell frequencies can be used for quantifying the surface topography similarity (or dissimilarity) between the KNM and KM image pairs, which addresses one of the requirements for a cell to be classified as a CMC. The additional congruency requirement will result in an average false cell identification probability for KNM comparisons that is equal to or lower than $f_{\text {1(ACCF). Alternatively, the }}$ average false cell exclusion probability for KM comparisons will be equal to or higher than $f_{2 \text { (ACCF). }}$. Therefore, low values of $f_{1 \text { (ACCF) }}$ and $f_{2 \text { (ACCF) }}$ provide improved conditions for the congruency evaluation to achieve low numbers of false positive or false negative CMCs. Thus, it is necessary to optimize $T_{\mathrm{ACCF}}$ so that the values of $f_{1(\mathrm{ACCF})}$ and $f_{2(\mathrm{ACCF})}$ can be well balanced. In our validation test, at each cell size, the kernel density estimation was used to fit $A C C F_{\max }$ distributions of KM and KNM cell pairs. Here,

$$
\hat{f}_{h}(x)=\frac{1}{n h} \sum_{i=1}^{n} K\left(\frac{x-x_{i}}{h}\right)
$$

where $x_{1}, x_{2}, \ldots, x_{\mathrm{n}}$ are random samples from an unknown distribution; $n$ is the sample size; $K$ is the kernel smoothing function, where the standard normal density function was used as the kernel function; and $h$ is the bandwidth, where $h$ was chosen based on the Silverman's rule of thumb [13].

$$
h=\left(\frac{4 \hat{\sigma}^{5}}{3 n}\right)^{\frac{1}{5}} \approx 1.06 \hat{\sigma} n^{-1 / 5}
$$

where $\hat{\sigma}$ is the standard deviation of the samples. The optimal $T_{\mathrm{ACCF}}$ was chosen at the intersection point of the two fit distributions as shown in Fig. 5 and listed in Table 1.

Table 1. Optimal $T_{\mathrm{ACCF}}$ and corresponding $P_{1(\mathrm{ACCF})}$ and $P_{2(\mathrm{ACCF})}$ values at each cell size for Fadul data set [10].

\begin{tabular}{ccccc}
\hline Cell size (pixels) & Cell numbers & Optimal $T_{\text {ACCF }}$ & $P_{1 \text { (ACCF) }}(\%)$ & $P_{\text {2(ACCF) }}(\%)$ \\
\hline $120 \times 120$ & $5 \times 5$ & 0.36 & 5.48 & 20.70 \\
$115 \times 115$ & $5 \times 5$ & 0.37 & 6.39 & 19.75 \\
$110 \times 110$ & $6 \times 6$ & 0.40 & 9.45 & 17.24 \\
$105 \times 105$ & $6 \times 6$ & 0.44 & 11.88 & 25.94 \\
$100 \times 100$ & $6 \times 6$ & 0.43 & 8.21 & 25.64 \\
$95 \times 95$ & $6 \times 6$ & 0.43 & 8.09 & 23.80 \\
$90 \times 90$ & $7 \times 7$ & 0.44 & 8.41 & 20.79 \\
$85 \times 85$ & $7 \times 7$ & 0.45 & 14.04 & 21.91 \\
$80 \times 80$ & $8 \times 8$ & 0.48 & 13.27 & 26.81 \\
$75 \times 75$ & $8 \times 8$ & 0.47 & 11.43 & 24.59 \\
$70 \times 70$ & $9 \times 9$ & 0.49 & 14.37 & 24.37 \\
$65 \times 65$ & $9 \times 9$ & 0.50 & 14.57 & 27.02 \\
$60 \times 60$ & $10 \times 10$ & 0.52 & 14.31 & 27.79 \\
$55 \times 55$ & $11 \times 11$ & 0.54 & 14.41 & 30.77 \\
$50 \times 50$ & $12 \times 12$ & 0.55 & 18.44 & 28.40 \\
$45 \times 45$ & $13 \times 13$ & 0.58 & 16.01 & 33.06 \\
$40 \times 40$ & $15 \times 15$ & 0.60 & 17.92 & 33.54 \\
$35 \times 35$ & $16 \times 16$ & 0.64 & 14.28 & 39.35 \\
$30 \times 30$ & $19 \times 19$ & 0.68 & 16.47 & 42.83 \\
$25 \times 25$ & $23 \times 23$ & 0.73 & 15.21 & 50.21 \\
$20 \times 20$ & $29 \times 29$ & 0.78 & 16.35 & 57.00 \\
$15 \times 15$ & $38 \times 38$ & 0.83 & 21.57 & 62.42 \\
\hline
\end{tabular}


Figure 6 shows the results using the CMC and NCMA methods at different cell sizes: $(75 \times 75)$ pixels and $(50 \times 50)$ pixels. As the cell size changes from $(75 \times 75)$ pixels to $(50 \times 50)$ pixels, the $\mathrm{CMC}$ distribution changes substantially, and the gap between the KM cell pair with the minimum CMCs and the KNM cell pair with the maximum CMCs changes from 9 to 17 . However, the gap between the KM cell pair with the minimum NCMA and the KNM cell pair with the maximum NCMA only changes from $29 \%$ to $27 \%$. The NCMA results show much more robustness with the change of cell size.
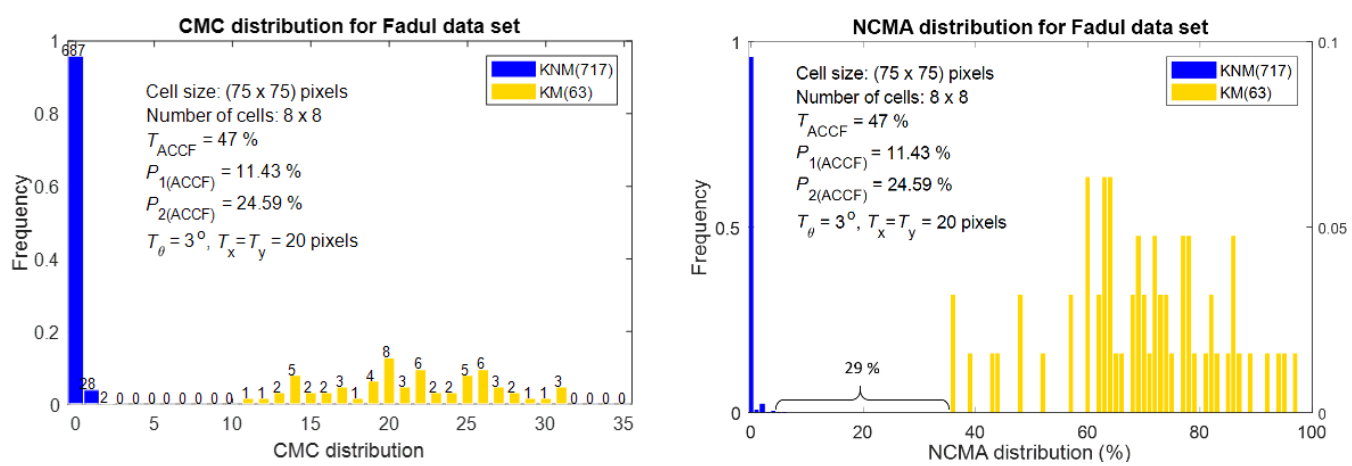

(a) Correlation results at cell size: $(75 \times 75)$ pixels $(6.25 \mu \mathrm{m}$ per pixel) $)$ using the CMC and NCMA methods.
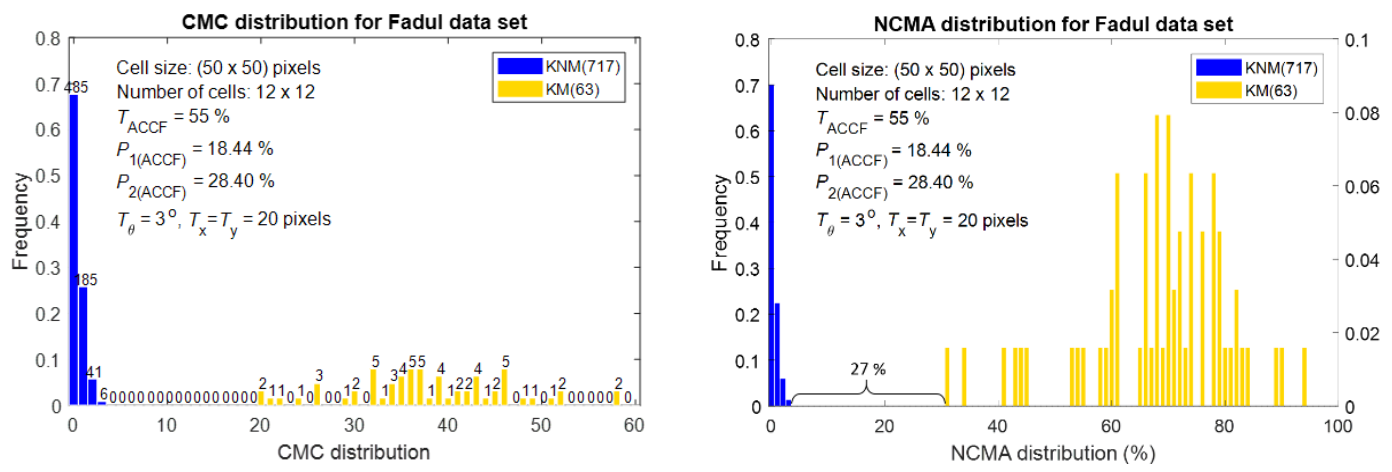

(b) Correlation results at cell size: $(50 \times 50)$ pixels $(6.25 \mu \mathrm{m}$ per pixel $)$ using the $\mathrm{CMC}$ and NCMA methods.

Fig. 6. CMC and NCMA correlation results for Fadul data set at different cell sizes using the CMC and NCMA methods.

Figure 7a shows the average NCMA value of the $63 \mathrm{KM}$ image pairs and $717 \mathrm{KNM}$ image pairs at different cell sizes from $(120 \times 120)$ pixels to $(15 \times 15)$ pixels with a pixel spacing of $6.25 \mu \mathrm{m}$. The average NCMA value of the KNM image pairs remains almost unchanged with the variation of cell size. However, the average NCMA value of KM image pairs changes with the cell size in a certain pattern: As the cell size decreases, the average NCMA value first fluctuates when the cell size is relatively large (around [100 $\times$ 100 ] pixels), then remains relatively steady (around [70 $\times 70]$ pixels), and finally drops rapidly (after [45 $\times$ 45] pixels). We split the KM average NCMA curve into three zones, the fluctuation zone, stable zone, and descending zone. To determine the boundaries of the three zones, a moving standard deviation of the KM average NCMA value was calculated, and it was plotted in Fig. 7a for analysis. Each point marked by a square in Fig. 7a is the standard deviation calculated over a sliding window of 7 neighboring elements of the KM average NCMA value. The smallest moving standard deviation appears at the cell size of $(65 \times 65)$ pixels, which means that the most stable region lies between $(50 \times 50)$ pixels and $(80 \times 80)$ pixels. So the fluctuation zone is from $(120 \times 120)$ pixels to $(80 \times 80)$ pixels (or $750 \mu \mathrm{m} \times 750 \mu \mathrm{m}$ to $500 \mu \mathrm{m} \times 500 \mu \mathrm{m}$, where the corresponding cell number is from $5 \times 5$ to $8 \times 8)$, the stable zone is from $(80 \times 80)$ pixels to $(50 \times 50$ ) pixels (or $500 \mu \mathrm{m} \times 500 \mu \mathrm{m}$ to $312.5 \mu \mathrm{m} \times 312.5 \mu \mathrm{m}$, where the corresponding cell number is from $8 \times 8$ to $12 \times 12$ ), and the descending zone is from $(50 \times 50)$ pixels to $(15 \times 15)$ pixels (or $312.5 \mu \mathrm{m} \times$ $312.5 \mu \mathrm{m}$ to $94 \mu \mathrm{m} \times 94 \mu \mathrm{m}$, where the corresponding cell number is from $12 \times 12$ to $38 \times 38$ ). 


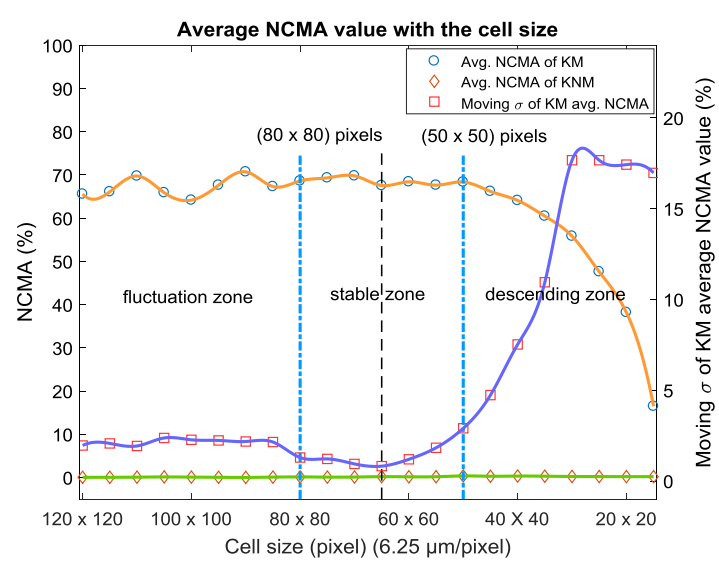

(a) Average NCMA value and the moving standard deviation of KM average NCMA value

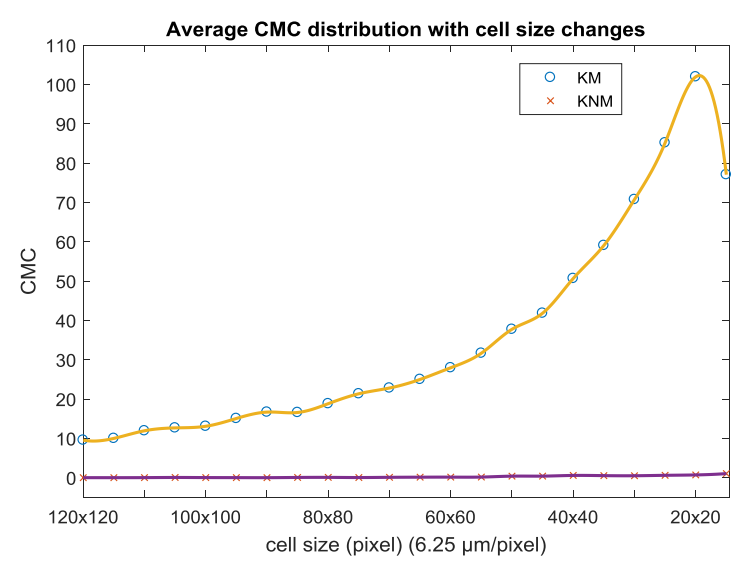

(b) Average CMC value

Fig. 7. The average NCMA and CMC values for the $63 \mathrm{KM}$ and $717 \mathrm{KNM}$ image pairs with the cell size changing from $(120 \times 120)$ pixels to $(15 \times 15)$ pixels $(6.25 \mu \mathrm{m}$ per pixel). The stable zone can be seen in NCMA method.

When the cell size is relatively large in the fluctuation zone, the valid and invalid correlation regions cannot be separated effectively by the cell, which may lead to either false identification or false exclusion, and finally demonstrates fluctuation in the average NCMA value. When the cell size gets smaller in the stable region, the cell is small enough to separate the valid and invalid correlation regions effectively, but not so small that it does not hold sufficient topography features. In this region, the curve remains stable and robust with changes in the cell size. When the cell size decreases further to the descending zone, the cell is too small to contain enough topography features for correlation, and some CMCs become invalid and are falsely excluded. Based on the results observed, we recommend a cell size range from about $500 \mu \mathrm{m} \times$ $500 \mu \mathrm{m}$ to $312.5 \mu \mathrm{m} \times 312.5 \mu \mathrm{m}$ (the corresponding cell number is $8 \times 8$ to $12 \times 12$ ) to be used in the firearm identification using breech face impression data with granular characteristics. This optimal cell size range is close to the size of topography features valid for identification.

\section{Test Results and a Robust Identification Criterion}

\subsection{Error Rate Estimation using the Conceptual Diagram of the NCMA Distribution}

Figure 7b shows that the CMC distribution of KM image pairs changes substantially with the cell size, which makes a fixed identification criterion such as $C=6$ (or other fixed value) not optimal or reliable when the cell size is extremely small or large. However, for the NCMA method, there exists a stable zone (see Fig. 7a, where the cell size ranges from about $500 \mu \mathrm{m} \times 500 \mu \mathrm{m}$ to $312.5 \mu \mathrm{m} \times 312.5 \mu \mathrm{m}$ ) where the NCMA distribution remains almost the same. Thus, an improved identification criterion can be determined from the KM and KNM population statistics and applied to the error rate estimation for false identifications and exclusions.

Theoretically, an experimental identification criterion can be defined at the cross-point $C(\%)$ of the $\mathrm{KM}$ and KNM distributions from the conceptual model in Fig. 8 [6, 7], where the NCMA probability mass functions, $\Phi_{\mathrm{NCMA}}$ and $\Psi_{\mathrm{NCMA}}$, of the $\mathrm{KM}$ and $\mathrm{KNM}$ topography pairs are equal. At this point, the scorebased likelihood ratio [14] $L R_{1}=L R_{2}=1$ :

$$
L R_{1}=\frac{\operatorname{Pr}\left(\Delta\left(X_{\mathrm{c}}, X_{\mathrm{s}}\right)=\delta \mid H_{\mathrm{p}}, I\right)}{\operatorname{Pr}\left(\Delta\left(X_{\mathrm{c}}, X_{\mathrm{s}}\right)=\delta \mid H_{\mathrm{d}}, I\right)}=\frac{\Phi_{(\mathrm{NCMA}=\delta)}}{\Psi_{(\mathrm{NCMA}=\delta)}}
$$




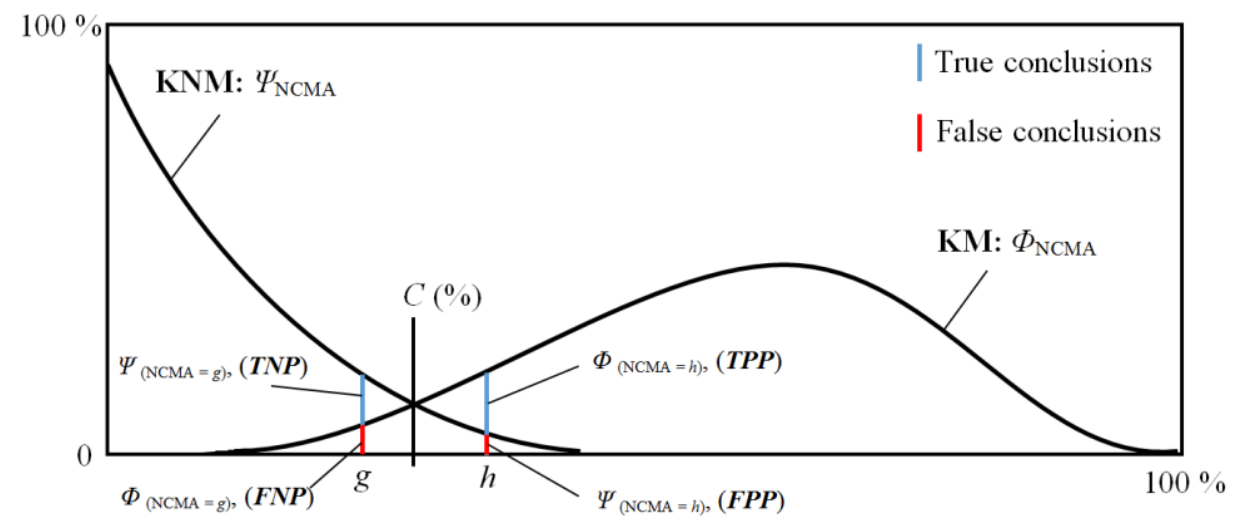

Fig. 8. Conceptual diagram of the NCMA probability mass functions of KM and KNM image correlations, $\Phi_{\mathrm{NCMA}}$ and $\Psi_{\mathrm{NCMA}}[6,7]$. TNP: true negative probability, FNP: false negative probability, TPP: true positive probability, FPP: false positive probability. The discrete probability distributions are shown as continuous density functions here. The overlapping area is much larger than they would be in practice for clarity.

$$
L R_{2}=\frac{\operatorname{Pr}\left(\Delta\left(X_{\mathrm{c}}, X_{\mathrm{s}}\right)=\delta \mid H_{\mathrm{d}}, I\right)}{\operatorname{Pr}\left(\Delta\left(X_{\mathrm{c}}, X_{\mathrm{s}}\right)=\delta \mid H_{\mathrm{p}}, I\right)}=\frac{\Psi_{(\mathrm{NCMA}=\delta)}}{\Phi_{(\mathrm{NCMA}=\delta)}}
$$

where

Pr: the probability value;

$\Delta$ : the NCMA process;

$X_{\mathrm{c}}$ : topography image of cartridge case from crime scene (here it is the reference image);

$X_{\mathrm{s}}$ : topography image of cartridge case from suspect gun (here it is the comparison image);

$\delta$ : NCMA score between the reference and comparison image;

$H_{\mathrm{p}}$ : the suspect firearm that fired the cartridge case found at the crime scene;

$H_{\mathrm{d}}$ : the suspect firearm that did not fire the cartridge case found at the crime scene; and

$I$ : background investigation information.

Given the data and assumptions used to model the relevant populations, the equation $L R_{1}=L R_{2}=1$ indicates that the result $C(\%)$ neither supports the hypothesis $H_{\mathrm{p}}$ over $H_{\mathrm{d}}$ nor support the hypothesis $H_{\mathrm{d}}$ over $H_{\mathrm{p}}$. With this criterion and the NCMA probability mass functions, $\Phi_{\mathrm{NCMA}}$ and $\Psi_{\mathrm{NCMA}}$, the potential error rates for cartridge cases fired from different source $(\mathrm{KNM})$ and same source $(\mathrm{KM})$ firearms can be estimated. For the NCMA distribution, although the NCMA value is expressed in percentage form, it is a discrete value and changes discontinuously, since the NCMA value is determined based on the relative data quantity in the CMCs. In this paper, all the NCMA values are rounded, and the minimum NCMA unit is $1 \%$. The cumulative false positive error rate $E_{1}$ is given by the sum of the discrete probability mass function values, $\Psi_{\mathrm{NCMA}}$, for NCMA values between $C(\%)$ and $100 \%[6,7]$ :

$$
E_{1}=\sum_{\mathrm{NCMA}=C}^{\mathrm{NCMA}=100 \%} \Psi_{(\mathrm{NCMA})}=\Psi_{(\mathrm{NCMA}=C)}+\Psi_{(\mathrm{NCMA}=C+1 \%)}+\ldots+\Psi_{(\mathrm{NCMA}=100 \%)}
$$

Similarly, the cumulative false negative error rate $E_{2}$ is given by the sum of the discrete probability mass function values, $\Phi_{\mathrm{NCMA}}$, for NCMA values between $0 \%$ and $(C[\%]-1 \%)[6,7]$ : 


$$
E_{2}=\sum_{\mathrm{NCMA}=0 \%}^{\mathrm{NCMA}=C-1 \%} \Phi_{(\mathrm{NCMA})}=\Phi_{(\mathrm{NCMA}=0 \%)}+\Phi_{(\mathrm{NCMA}=1 \%)}+\ldots+\Phi_{(\mathrm{NCMA}=C-1 \%)}
$$

\subsection{Statistical Models for the NCMA Distribution}

The NCMA probability mass functions of KM and KNM image correlations, $\Phi_{\mathrm{NCMA}}$ and $\Psi_{\mathrm{NCMA}}$, are essential for the above calculation. Inspired by the statistical fitting process in Ref. [7], a binomial model was used to fit the KNM NCMA distribution $\Psi_{\mathrm{NCMA}}$, and a beta-binomial model was used to fit the KM NCMA distribution $\Phi_{\mathrm{NCMA}}$.

For the KNM NCMA distribution, if we consider $1 \%$ data quantity of the breech face impression area in the reference image as one unit, then this unit should be considered "congruent matching" with the comparison image (or be qualified as $1 \%$ NCMA) randomly and nonselectively. This theoretically meets the assumptions of binomial distributions: (1) For each image pair, whether the units on the reference image are found "congruent matching" with the comparison image can be considered as independent Bernoulli trials; (2) the unit trial success probability $p_{\mathrm{KNM}}$ is the same for all unit trials within each image pair. Then, the NCMA probability mass functions of KNM image correlations, $\Psi_{\mathrm{NCMA}}$, with a NCMA value $h$ can be calculated as:

$$
\Psi_{(\mathrm{NCMA}=h)}=C_{N}^{h} \cdot \hat{p}_{\mathrm{KNM}}^{h} \cdot\left(1-\hat{p}_{\mathrm{KNM}}\right)^{N-h}
$$

Here, we suppose for a KNM image correlation that the total data quantity of the breech face impression in consideration is $100 \%$, which means $N=100$. Also, $\hat{p}_{\mathrm{KNM}}$ is the maximum likelihood estimation of $p_{\mathrm{KNM}}$ from the observed KNM NCMA distribution.

However, for the KM NCMA distribution, the situation is more complicated. Since the toolmarks on the firearm breech face are affected by the firing conditions, contaminants, corrosion, etc., a mixture of valid and invalid correlation areas exists in the cartridge case breech face impression region. In addition, the quality of the toolmarks imparted in the common valid correlation area of the KM image pairs may differ anywhere. These two main factors will cause variations in the probability $p_{\mathrm{KM}}$ of the units (one unit is $1 \%$ data quantity) of the breech face impression area in the reference image being qualified as $1 \%$ NCMA with the comparison image.

In this approach, we still assume that: (1) the NCMA image correlation can be considered as a set of independent Bernoulli trials similar to the KNM NCMA distribution. However, (2) the unit trial success probability $p_{\text {Км }}$ is now modeled as a random variable with a beta distribution. The beta distribution is a continuous distribution on the interval $[0,1]$, and it has a variety of distribution shapes with shape parameters $\alpha>0$ and $\beta>0$ [15]. The beta distribution is also a conjugate distribution of the binomial distribution in Bayesian statistics [16], which makes the joint beta-binomial distribution easily evaluated and mathematically convenient [17]. The NCMA probability mass functions of KM image correlations, $\Phi_{\mathrm{NCMA}}$, with a NCMA value $h$ can be calculated as:

$$
\begin{gathered}
\Phi_{(\mathrm{NCMA}=h)}=C_{N}^{h} \cdot p_{\mathrm{KM}}^{h} \cdot\left(1-p_{\mathrm{KM}}\right)^{N-h} \\
f\left(p_{\mathrm{KM}} \mid \alpha, \beta\right)=\frac{p^{\alpha-1}(1-p)^{\beta-1}}{B(\alpha, \beta)}
\end{gathered}
$$

and then the compound beta-binomial distribution is: 


$$
\Phi_{(\mathrm{NCMA}=h)}=C_{N}^{h} \cdot \frac{B(h+\alpha, N-h+\beta)}{B(\alpha, \beta)}
$$

where $N$ still equals 100 , and $B(\alpha, \beta)$ is a beta function with parameters $\alpha$ and $\beta$. The maximum likelihood estimations $\hat{\alpha}$ and $\hat{\beta}$ are used in practice from the observed KM distribution data to replace $\alpha$ and $\beta$ in Eq. (8) [18].

\subsection{Experimental Identification Criteria $C$ for the NCMA Method}

The binomial model is used to fit the KNM NCMA probability distribution $\Psi_{\mathrm{NCMA}}$, and the betabinomial model is used to fit the KM NCMA probability distribution $\Phi_{\mathrm{NCMA}}$. The experimental identification criterion $C$ is determined at the intersection point of the two distributions. Figure 9 shows the NCMA distributions and the corresponding criteria $C$ for the Fadul data set at two different cell sizes within the optimal cell size range recommended in Sec. 3. The identification criteria $C$ remains $6 \%$ at the cell size of $(65 \times 65)$ pixels and $(55 \times 55)$ pixels. The little change in the experimental criteria $C$ indicates the NCMA distributions are very robust, even with the change in cell size.
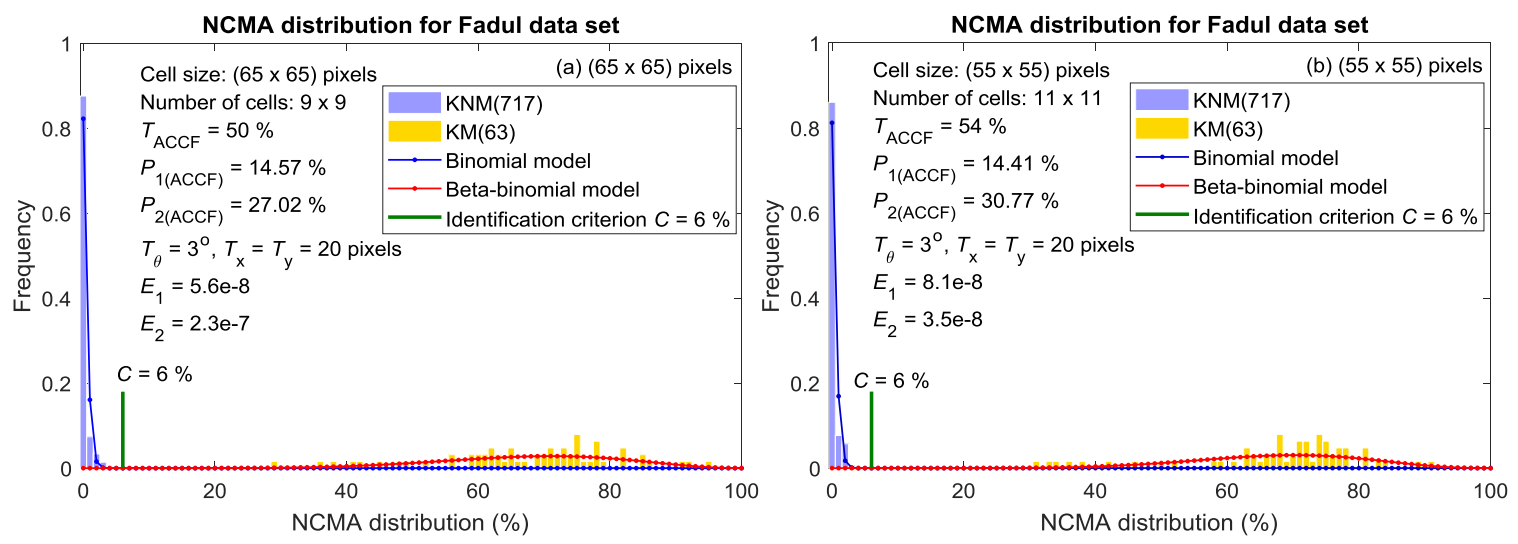

Fig. 9. The NCMA distributions for Fadul data set and the corresponding experimental identification criteria $C$ at cell sizes of (a) $(65 \times$ $65)$ pixels and (b) $(55 \times 55)$ pixels $(6.25 \mu \mathrm{m}$ per pixel). The solid blue curves represent the binomial distribution model for the KNM data, and the solid red curves represent the beta-binomial distribution model for the KM data.

\section{Discussion and Conclusion}

In the original CMC method, the final CMC scores are greatly influenced by the cell size, which in turn makes a fixed criterion for CMC method not optimal or reliable when the cell size is extremely large or small. A normalized congruent matching area (NCMA) method is proposed based on the difference in the data quantity (or validity level) of cells. The CMC numbers can be transformed into an NCMA value in a percentage form, which is more compatible with the actual situation, i.e., how much congruent matching area the two samples have. A validation test using the Fadul data set at cell sizes from $(120 \times 120)$ pixels to $(15 \times 15)$ pixels with an increment of 5 pixels demonstrates that the average NCMA value of KNM image pairs remains almost the same at all cell sizes, while that of KM image pairs first fluctuates, then remains stable, and finally drops rapidly as the cell sizes change from large to small. On the contrary, no stable region can be found in the average CMC curve of KM image pairs. The stable region is recommended as an optimal cell size range, which is from about $500 \mu \mathrm{m} \times 500 \mu \mathrm{m}$ to $312.5 \mu \mathrm{m} \times 312.5 \mu \mathrm{m}$ for breech face 
identification with granular characteristics - this cell size is small enough to separate the valid and invalid correlation regions effectively, but it still holds enough topography features in this cell size range.

Taking advantage of the stability of the NCMA distribution at the optimal cell size range, an experimental robust identification criterion $C$ is established at the intersection point of the KM and KNM NCMA probability mass functions $\Phi_{\mathrm{NCMA}}$ and $\Psi_{\mathrm{NCMA}}$, where the score-based likelihood ratio $L R_{1}=L R_{2}=$ 1 . The $\Phi_{\mathrm{NCMA}}$ and $\Psi_{\mathrm{NCMA}}$ functions are fitted by beta-binomial and binomial models, respectively. Results at cell sizes of $(65 \times 65)$ pixels and $(55 \times 55)$ pixels show that the current identification criterion $C$ barely changes with the cell size, indicating that the NCMA distributions are very robust in the optimal cell size range, and robust optimal identification criteria are available for the NCMA method.

\subsection{Future Work}

The statistical model fitting in Sec. 4.3 of this manuscript was mainly based on Ref. [7]. However, after the submission of this manuscript, Nien Fan Zhang, an author of Ref. [7], had a breakthrough for the statistical model fitting in estimating error rates for firearm evidence identifications. A beta-correlated binomial model is proposed in his new manuscript [19]. The beta-correlated binomial model not only relaxes the assumption for the same cell pair identification probability, but it relaxes the assumption of independence among the cell pair comparisons as well. This model is expected to have potentially better fits for the CMC measurements than the existing models, and it is considered an upgrade and supplement for the current NIST error rate estimation suite for ballistics identification. Considering the unique feature that this model can relax the assumption of independence, we plan to apply this newly developed model in our future research, especially for the correlation with the presence of "patches" of subclass characteristics.

\section{Acknowledgments}

The funding for this work was provided by the Special Programs Office (SPO) of NIST. The authors are grateful to D. Ott of NIST for assistance with the algorithm and test design; to X. A. Zheng of NIST for providing the topography images; to X. A. Zheng and Q. An of NIST for their review and helpful comments on the manuscript; and to R. M. Thompson of NIST for his insights on ballistics identification.

\section{References}

[1] Scientific Working Group for Firearm and Toolmarks (SWGGUN) (2013) The Foundations of Firearm and Toolmark Identification. Available at https://www.nist.gov/sites/default/files/documents/2016/11/28/swggun_foundational_report.pdf. Accessed July 31, 2018.

[2] Song J, Chu W, Tong M, Soons J (2014) 3D topography measurements on correlation cells-A new approach to forensic ballistics identifications. Measurement Science and Technology 25(6):064005. https://doi.org/10.1088/0957-0233/25/6/064005

[3] Song J (2013) Proposed NIST Ballistics Identification System (NBIS) using 3D topography measurements on correlation cells. Journal of the Association of Firearms and Toolmarks Examiners 45(2):184-194.

[4] Chu W, Tong M, Song J (2013) Validation tests for the congruent matching cells (CMC) method using cartridge cases fired with consecutively manufactured pistol slides. Journal of the Association of Firearms and Toolmarks Examiners 45(4):361-366.

[5] Tong M, Song J, Chu W, Thompson RM (2014) Fired cartridge case identification using optical images and the congruent matching cells (CMC) method. Journal of Research of the National Institute of Standards and Technology 119:575. https://doi.org/10.6028/jres.119.023

[6] Song J (2015) Proposed "congruent matching cells (CMC)" method for ballistic identification and error rate estimation. Journal of the Association of Firearms and Toolmarks Examiners 47(3):177-185.

[7] Song J, Vorburger TV, Chu W, Yen J, Soons JA, Ott DB, Zhang NF (2018) Estimating error rates for firearm evidence identifications in forensic science. Forensic Science International 284:15-32. https://doi.org/10.1016/j.forsciint.2017.12.013

[8] Tong M, Pan Y, Li Z, Lin W (2018) Valid data based normalized cross-correlation (VDNCC) for topography identification. Neurocomputing, 308:184-193. https://doi.org/10.1016/j.neucom.2018.04.059 
[9] Biasotti A, Murdock J (1997) Firearms and toolmark identification: Legal issues and scientific status. Modern Scientific Evidence: The Law and Science of Expert Testimony, eds Faigman D, Kaye D, Saks M, Sanders J (West Publishing Co., St. Paul, MN), pp 124-151.

[10] Fadul TG Jr, Hernandez GA, Stoiloff S, Gulati S (2012) An Empirical Study to Improve the Scientific Foundation of Forensic Firearm and Tool Mark Identification Utilizing 10 Consecutively Manufactured Slides (National Institute of Justice, Washington, D.C.), NIJ Report No. 237960. Available at https://www.ncjrs.gov/pdffiles1/nij/grants/237960.pdf. Accessed July 31, 2018.

[11] Hamilton DK, Wilson T (1982) Three-dimensional surface measurement using the confocal scanning microscope. Applied Physics 27(4):211-213. https://doi.org/10.1007/BF00697444

[12] Silverman BW (2018) Density Estimation for Statistics and Data Analysis (Chapman \& Hall, Boca Raton).

[13] Bolck A (2016) Score-based and Feature-based LRs. Workshop on Theory and Implementation of Numerical Likelihood Ratio Methods, (Netherlands Forensic Institute (NFI), The Hague, Netherlands), May 23-24, 2016.

[14] Falls LW (1974) The beta distribution: A statistical model for world cloud cover. Journal of Geophysical Research 79(9):12611264. https://doi.org/10.1029/JC079i009p01261

[15] Diaconis P, Ylvisaker D (1979) Conjugate priors for exponential families. The Annals of Statistics 7(2):269-281. https://www.jstor.org/stable/2958808

[16] Chen JJ, Novick MR (1984) Bayesian analysis for binomial models with generalized beta prior distributions. Journal of Educational and Behavioral Statistics 9(2):163-175. https://www.jstor.org/stable/1164719

[17] Smith DM (1983) Algorithm AS 189: Maximum likelihood estimation of the parameters of the beta binomial distribution. Journal of the Royal Statistical Society Series C (Applied Statistics) 32(2):196-204. https://www.jstor.org/stable/2347299

[18] Zhang NF (Submitted) The use of correlated binomial distributions in estimating error rates for firearm evidence identifications. To be submitted to Journal of the Royal Statistical Society Series C (Applied Statistics).

[19] Chen Z, Song J, Chu W, Soons JA, Zhao X (2018) A convergence algorithm for correlation of breech face images based on the congruent matching cells (CMC) method. Forensic Science International 280:213-223. https://doi.org/10.1016/j.forsciint.2017.08.033

About the authors: Zhe Chen is a guest researcher in the Surface and Nanostructure Metrology Group (SNMG) of the Engineering Physics Division (EPD) of the Physical Measurement Laboratory (PML) at NIST. John Song is a project leader and postdoctoral research advisor for the Forensic Topography and Surface Metrology Project of the SNMG. Wei Chu is a contractor of the Forensic Topography and Surface Metrology Project of SNMG. Mingsi Tong is a researcher in mechatronics engineering at Harbin Institute of Technology (HIT). XueZeng Zhao is a professor of mechatronics engineering at Harbin Institute of Technology (HIT).

The National Institute of Standards and Technology (NIST) is an agency of the U.S. Department of Commerce. 